IASSNS-HEP-94/27

\title{
Exact Dynamical Correlation Functions of Calogero-Sutherland Model and One-Dimensional Fractional Statistics
}

\author{
Z. N. C. Ha \\ School of Natural Sciences, Institute for Advanced Study, \\ Princeton, New Jersey 08540
}

(April 18, 1994)

\begin{abstract}
One-dimensional (1D) model of non-relativistic particles with inverse-square interaction potential known as Calogero-Sutherland Model (CSM) is shown to possess fractional statistics. Using the theory of Jack symmetric polynomial the exact dynamical density-density correlation function and the one-particle Green's function (hole propagator) at any rational interaction coupling constant $\lambda=p / q$ are obtained and used to show clear evidences of the fractional statistics. Motifs representing the eigenstates of the model are also constructed and used to reveal the fractional exclusion statistics (in the sense of Haldane's "Generalized Pauli Exclusion Principle"). This model is also endowed with a natural exchange statistics (1D analog of $2 \mathrm{D}$ braiding statistics) compatible with the exclusion statistics.
\end{abstract}

(Submitted to PRL on April 18, 1994)

PACS 05.30-d, 71.10.+x

Typeset using REVTEX 
The quantum particles obeying fractional statistics known as anyons have been a subject of intense study since the discovery of the fractional quantum Hall effect and the High $T_{c}$ super-conductors [1]. The subject, however, is far from complete. In fact, even the simplest model of anyons, namely the ideal gas, has not yet been fully understood. To add to the confusion, there are now two seemingly non-equivalent definitions of fractional statistics. While the popular definition of anyons is based on the quantum phase arising from the exchange of particles [1], Haldane's definition [2] is based on so called the "Generalized Pauli Exclusion Principle." The main difference in the two approach is that while in the former the statistics is assigned to the Newtonian point particles, in the latter it is obeyed by the elementary excitations of condensed matter system.

There are no fully solvable two-dimensional models where the ideas of fractional statistics can be rigorously tested. In one-dimension, however, there is such a model known as Calogero-Sutherland Model (CSM) [3]. I show in this Letter that the two definitions of fractional statistics can coexist in CSM without any inconsistency. Following Ref. [4], I construct the motifs for all the excited states and explicitly demonstrate that the quasi-particles and quasi-holes obey the Haldane's exclusion statistics. I also solve the exact ground state dynamical density-density correlation function (DDDCF) and the hole propagator part of the one-particle Green's function (HPOGF) and show that the contributing intermediate states involve only a finite number of quasi-particles (holes) consistent with the ideal "anyon" gas structure of this model. In calculating the correlation function and propagator a new mathematical technique based on the theory of Jack symmetric orthogonal polynomial [5] is used.

The CSM Hamiltonian, which describes a system of $N$ non-relativistic particles interacting with inverse-square exchange, is given by

$$
H=-\sum_{j=1}^{N} \frac{\partial^{2}}{\partial x_{j}^{2}}+\sum_{j<l} \frac{2 \lambda(\lambda-1)}{d^{2}\left(x_{j}-x_{l}\right)},
$$

where $\hbar^{2} / 2 m=1$ and $d\left(x_{i}-y_{j}\right)$ is the chord distance between the $i$ th and $j$ th particles on a ring of length $L$, and is equal to $\left|(L / \pi) \sin \left(\pi\left(x_{i}-y_{j}\right) / L\right)\right|$. The dimension-less interaction 
coupling constant $\lambda$ is a positive real number that specifies the natural statistics of this model. For the special values of $\lambda=1 / 2,1$, and 2 , the model is related to the orthogonal, unitary and simplectic random matrix theory [6], respectively, and DDDCF for those values have previously been found by the supersymmetry technique [7]. For $\lambda=2$, the full oneparticle Green's function has also been found [8,9].

One of the main results of this Letter is the calculation of the dynamical density-density correlation function at any rational interaction parameter $\lambda=p / q$. It is given by

$$
\langle 0|\rho(x, t) \rho(0,0)| 0\rangle=C \operatorname{Re} \prod_{i=1}^{q}\left(\int_{0}^{\infty} d x_{i}\right) \prod_{j=1}^{p}\left(\int_{0}^{1} d y_{j}\right) Q^{2} F\left(q, p, \lambda \mid\left\{x_{i}, y_{j}\right\}\right) e^{i(Q x-E t)},
$$

where $Q$ (momentum) and $E$ (energy) are given by

$$
\begin{aligned}
& Q=2 \pi \rho\left(\sum_{j=1}^{q} x_{j}+\sum_{j=1}^{p} y_{j}\right) \\
& E=(2 \pi \rho)^{2}\left(\sum_{j=1}^{q} \epsilon_{P}\left(x_{j}\right)+\sum_{j=1}^{p} \epsilon_{H}\left(y_{j}\right)\right),
\end{aligned}
$$

where $\rho=N / L, \epsilon_{P}(x)=x(x+\lambda)$ and $\epsilon_{H}(y)=\lambda y(1-y) . x_{j}\left(\epsilon_{P}\right)$ and $y_{j}\left(\epsilon_{H}\right)$ are normalized momentum (energy) of the quasi-particles and the quasi-holes, respectively. The normalization constant $C$ is given by

$$
\begin{aligned}
A(m, n, \lambda) & =\frac{\Gamma^{m}(\lambda) \Gamma^{n}(1 / \lambda)}{\prod_{i=1}^{m} \Gamma^{2}(p-\lambda(i-1)) \prod_{j=1}^{n} \Gamma^{2}(q-(j-1) / \lambda)} \prod_{j=1}^{n}\left(\frac{\Gamma(q-(j-1) / \lambda)}{\Gamma(1-(j-1) / \lambda)}\right)^{2} \\
C & =\frac{\lambda^{2 p(q-1)} \Gamma^{2}(p)}{2 \pi^{2} p ! q !} A(q, p, \lambda) .
\end{aligned}
$$

Finally, the form factor $F\left(q, p, \lambda \mid\left\{x_{i}, y_{j}\right\}\right)$ is given by

$$
F\left(q, p, \lambda \mid\left\{x_{i}, y_{j}\right\}\right)=\prod_{i=1}^{q} \prod_{j=1}^{p}\left(x_{i}+\lambda y_{j}\right)^{-2} \frac{\left(\prod_{i<j}\left(x_{i}-x_{j}\right)^{2}\right)^{\lambda}\left(\prod_{i<j}\left(y_{i}-y_{j}\right)^{2}\right)^{1 / \lambda}}{\prod_{i=1}^{q} \epsilon_{P}\left(x_{i}\right)^{1-\lambda} \prod_{j=1}^{p} \epsilon_{H}\left(y_{j}\right)^{1-1 / \lambda}} .
$$

The results of Simons, et al. at $\lambda=1 / 2,1$ and 2 with an appropriate change of variables agree with Eq. (2) up to the normalization constant. Recently, based on a mapping to 2D QCD Minahan guessed correctly the form factor for the integer values of $\lambda$ and their duals $1 / \lambda[10$. Haldane also guessed the correct form factor for the rational $\lambda=p / q$ based on the 
information given by Simons, et al., Galilean invariance, and asymptotic agreement with $\mathrm{U}(1)$ conformal field theory 11 .

The ground state of SCM is given by

$$
\Psi_{0}=\prod_{j>l}\left(z_{j}-z_{l}\right)^{\lambda} \prod_{j=1}^{N} z_{j}^{J_{0}}
$$

where $z_{j}=\exp \left(i 2 \pi x_{j} / L\right)$ and $J_{0}=-\lambda(N-1) / 2$. If a general eigenfunction with energy $E$ is written as $\Psi=\Psi_{0} \Phi$, then $\Phi$ is an eigenstate of the following new effective Hamiltonian $H^{\prime}$

$$
H^{\prime} \Phi=\sum_{i}\left(z_{i} \partial_{z_{i}}\right)^{2} \Phi+\lambda \sum_{i<j} \frac{z_{i}+z_{j}}{z_{i}-z_{j}}\left(z_{i} \partial_{z_{i}}-z_{j} \partial_{z_{j}}\right) \Phi=\varepsilon \Phi
$$

where $\varepsilon=(L / 2 \pi)^{2}\left(E-E_{0}\right)$. An amazing coincidence happens if $\Delta=(\lambda N-\lambda-1) \sum_{j} z_{j} \partial_{z_{j}}$ is added to Eq. (9). A complete set of linearly independent solution of the resulting equation is known in mathematical literature as the Jack polynomial $J_{\kappa}^{1 / \lambda}\left(z_{1}, \ldots, z_{N}\right)$ [5]. The index $\kappa=\left(\kappa_{1}, \kappa_{2}, \ldots, \kappa_{N}\right)$ is partition of non-negative integers and is essentially a set of bosonic quantum numbers used to label all the eigenstates of SCM up to global Galilean boosts. In particular, the ground state is given by the partition of zero (i.e. $\kappa_{1}=\kappa_{2}=\ldots=\kappa_{N}=0$ ). The parts $\kappa_{j}$ 's of partition $\kappa$ are ordered so that $\kappa_{1} \geq \kappa_{2} \geq \cdots \geq \kappa_{N}$. Since the $J_{\kappa}$ is a homogeneous symmetric polynomial of degree $|\kappa|=\sum_{j} \kappa_{j}$, it is also an eigenfunction of $\Delta$ with eigenvalue $(\lambda N-\lambda-1)|\kappa|$ and thus of $H^{\prime}$ with $\varepsilon=\sum_{j} \kappa_{j}+\lambda(N+1-2 j) \kappa_{j}$. If the pseudo-momenta $k_{j}$ 's are defined by

$$
L k_{j}=2 \pi I_{j}+\pi(\lambda-1) \sum_{l} \operatorname{sgn}\left(k_{j}-k_{l}\right)
$$

with $I_{j}=\kappa_{j}+(N+1-2 j) / 2$, then the eigenenergy and momentum take the following free form: $E=\frac{\hbar^{2}}{2 m} \sum_{j} k_{j}^{2}$ and $P=\sum_{j} k_{j}$. Using a method developed by Yang and Yang [12], Sutherland calculated the thermodynamics for SCM [13] and found that the densities of occupied $\left(\rho_{P}\right)$ and unoccupied $\left(\rho_{H}\right) k$ satisfy the following relation: $\lambda \rho_{P}(k)+\rho_{H}(k)=1$. This is a statement of broken particle-hole symmetry for $\lambda \neq 1$ and an analog of the ChernSimons duality. $\rho_{P}(k)$ satisfies the following relation [14], $\left(1-\lambda \rho_{P}\right)^{\lambda}\left(1-(\lambda-1) \rho_{P}\right)^{\lambda-1}=$ 
$\rho_{P} \exp ((\epsilon(k)-\mu) / T)$ with $\epsilon(k)=k^{2}$, which is identical to that of the ideal gas obeying Haldane's fractional exclusion statistics [15]. I emphasize here that the statistical distribution above is only for the pseudo-particles and is not related to the statistics of the real particles.

I use three different names for the particles in the model-real, pseudo-, and quasiparticles. The real particles are, of course, the physical quantum particles described by the canonically conjugate coordinate and momentum variables $\left\{x_{j}, p_{j}\right\}$. The pseudo-particles are described by the pseudo-momentum operators (see Eq. (15)) whose eigenvalues are given by Eq. (10). The quasi-particles are the elementary excitations of the system. Because the pseudo-particles form an ideal gas, the quasi-particles are essentially same as the pseudoparticles excited out of the condensate. The holes left behind in the pseudo-particle condensate will be called quasi-holes. Hence, the name "pseudo" and "quasi" will be used interchangeably in some cases.

If $\lambda=p / q$, where $p$ and $q$ are relative primes, the motifs for eigenstates can be constructed. The ones and zeroes in the motif mean occupied and unoccupied $k_{j}$, respectively. From Eq. (10) the following rules can be deduced for constructing the motif. (i) Total of $N$ ones in the motif represent the pseudo-particles. Hence, the charge of pseudo- and real particle is same. (ii) The allowed number of zeroes between each pair of ones is $p-1+n q$, where $n$ is an arbitrary non-negative integer. Of the $p-1+n q$ allowed zeroes, $p-1$ consecutive zeroes are bound to each one while the rest of them are un-bound. The consecutive $q$ un-bound zeroes represent a hole. (iii) A cutoff is introduced to make the number of unbound zeroes finite. The natural unit for the pseudo-momenta is then $2 \pi / q L$ which is an analog of the flux quantum. To give some examples, the ground state $(a)$, allowed $(b)$ and forbidden $(c)$ states for $\lambda=3 / 4$ for $N=7$ are represented, respectively, by

$$
\begin{aligned}
& \text { (a) } \cdots 00000000100100100100100100100000000 \cdots \\
& \text { (b) } \cdots 00000000100100100100100100000010000 \cdots \\
& \text { (c) } \cdots 00000000100100100000100100100100000 \cdots
\end{aligned}
$$

The motif (c) violates Rule (ii) and hence not allowed. The bound zeros which cause spacings 
between the ones can be viewed as flux attached to the pseudo-particles.

The motif thus constructed is a powerful tool in visualizing the fractional statistics. Destroying a particle (or turning a one into a zero) creates $p$ extra un-bound zeroes and in order to have integer number of holes in the condensate (Rule (ii)), minimum of $q-1$ extra quasi-particles must be excited out of the condensate leaving behind total of $q p$ unbound zeroes which break up into $p$ holes. Hence, $q$ particles leave behind $p$ holes. This is a generalization of the Pauli exclusion principle which explains intuitively why the densitydensity correlation function has $q$ quasi-particles and $p$ quasi-holes when $\lambda=p / q$. It will be shown explicitly that the only states that contribute to the DDDCF are indeed this minimal excitation.

Before I begin to discuss the method used to calculate the DDDCF, some notations need to be defined. First, a diagram $\mathcal{D}(\kappa)$ is defined to be rows and columns of boxes labeled by $\left\{(i, j): 1 \leq i \leq l(\kappa), 1 \leq j \leq \kappa_{i}\right\}$, where $l(\kappa)$ denotes the number of non-zero $\kappa_{j}$. The label $i$ and $j$ are row and column indices of the diagram with $l(\kappa)$ rows of lengths $\kappa_{j}$. Second, the conjugate of $\kappa$ denoted by $\kappa^{\prime}$ is obtained from $\kappa$ by changing all the rows to columns in nonincreasing order [16]. Each row (column) corresponds to the quasi-particle (hole) excitations. Third, a generalization of factorial is defined by $[a]_{\kappa}^{\lambda}=\prod_{(i, j) \in \kappa}(a+(j-1) / \lambda-(i-1))$. Using the above notations, the density-density correlation function at finite $N$ and $L$ is given by

$$
\langle 0|\rho(x, t) \rho(0,0)| 0\rangle=\frac{2}{\lambda^{2}} \sum_{\kappa} \frac{|\kappa|^{2}}{j_{\kappa}^{\lambda}} \frac{\left(\left[0^{\prime}\right]_{\kappa}^{\lambda}\right)^{2}[N]_{\kappa}^{\lambda}}{[N+1 / \lambda-1]_{\kappa}^{\lambda}} e^{i 2 \pi|\kappa| x / L} e^{-i t E_{\kappa}},
$$

where $E_{\kappa}=\sum_{j=1}^{N} \kappa_{j}^{2}+\lambda \sum_{j=1}^{N}(N+1-2 j) \kappa_{j}$ and $j_{\kappa}^{\lambda}=\prod_{(i, j) \in \kappa}\left(\kappa_{j}^{\prime}-i+1+\left(\kappa_{i}-j\right) / \lambda\right)\left(\kappa_{j}^{\prime}-\right.$ $\left.i+\left(\kappa_{i}-j+1\right) / \lambda\right)$. The product in $\left[0^{\prime}\right]_{\kappa}^{\lambda}$ does not include the pair $(i, j)=(1,1)$. In deriving the finite size correlation function, I use the following two relations [17, 18

$$
\begin{aligned}
\sum_{i} z_{i}^{n} & =\frac{n}{\lambda} \sum_{|\kappa|=n} \frac{\left[0^{\prime}\right]_{\kappa}^{\lambda}}{j_{\kappa}^{\lambda}} J_{\kappa}^{1 / \lambda}\left(\left\{z_{i}\right\}\right), \\
\left\langle\kappa \mid \kappa^{\prime}\right\rangle_{\lambda} & =j_{\kappa}^{\lambda} \frac{[N]_{\kappa}^{\lambda}}{[N+1 / \lambda-1]_{\kappa}^{\lambda}} \delta_{\kappa, \kappa^{\prime}} .
\end{aligned}
$$

The first relation can be used to expand the density operator $(1 / L) \sum_{j=1}^{N} \delta\left(x-x_{j}\right)$ in terms of the Jack symmetric polynomial. The second relation gives the normalization constants 
of all the excited states parameterized by $\kappa$. The DDDCF is now easy to calculate since the eigenstate $\kappa$ evolves in time only with a phase $\exp \left(-i t E_{\kappa}\right)$.

For $\lambda=p / q$, the coefficient $\left[0^{\prime}\right]_{\kappa}^{\lambda}$ is zero if $\mathcal{D}(\kappa)$ consists of more than $p$ columns or more than $q$ rows. Hence, in the thermodynamic limit a local density operator acting on the ground state provokes only the minimal excitations consisting of $q$ and $p$ quasi-particles and holes.

The large $N$ expansion of Eq. (11) can be carried out at fixed density $\rho=N / L$ and the leading order term corresponding to the thermodynamic limit is given by Eq. (2). In this limit, a new super selection rule emerges and suppresses the states with $\left|\kappa_{i}-\kappa_{j}\right| \approx O(1)$ to $O\left(1 / N^{2 \lambda}\right)$ or $O\left(1 / N^{2 / \lambda}\right)$ depending on the value of $\lambda$. This means that the states with quasi-particles and quasi-holes with same momenta (velocities) are suppressed. There are some exotic exceptions to this rule. The details will be published elsewhere.

The form of the ground state wave-function, Eq. (8), has lead Haldane to suggest that while the apparent statistics can be modified with a singular gauge transformation, the "natural" statistics of the SCM are fractional, and that the particle excitations carry charge 1 and flux $\pi \lambda$ and the hole excitations charge $-1 / \lambda$ and flux $-\pi$ [19]. Indeed, if a singular gauge transformation [20] is applied, the ground state wave function can be rewritten as

$$
\Psi_{0}=\prod_{j>l} \frac{\left(z_{j}-z_{l}\right)^{\beta}}{\left|z_{j}-z_{l}\right|^{\beta}}\left|z_{j}-z_{l}\right|^{\lambda} \prod_{k} z_{k}^{-\alpha(N-1) / 2},
$$

where the apparent statistical parameter is now $\theta=\pi \beta$. Unlike in the two-dimensional case, the transformed wave function remains as the ground state of the original Hamiltonian and so are all the other eigenstates. The DDDCF is also unchanged. The HPOGF, however, will be different for different choices of statistics (see, for example, [21]). Hence, in order to calculate the HPOGF, it will be necessary to adapt Haldane's "natural" exchange statistics for the SCM particles.

In order to consider the fractional exchange statistics, it is convenient to multiply the wave function $\Psi$ by an "ordering function" $\varphi\left(x_{P_{1}}, \ldots, x_{P_{N}}\right)$ which is just a book keeping device for the phase factor that depends on the permutation $P$ and is set to unity for the 
fundamental region $x_{1}<x_{2}<\cdots<x_{N}$. (In the case of Fermions the function is just a product of Grassmann numbers.) Since the ordering function automatically keeps track of all the exchange phases, the particle exchange operator $P_{i j}$ acting on the full wave function amounts to simply exchanging the indices $i$ and $j$. If the "natural" statistics is chosen, the phases arising from $\varphi$ and $\Psi$ can always be set to cancel each other!

In the original SCM there is no physical process which allows particles to exchange. (i.e. The wave function vanishes like $\left|x_{i}-x_{j}\right|^{\lambda}$ as $x_{i} \rightarrow x_{j}$.) A few years ago, Polychronakos 22 solved this problem by introducing an analog of the Yangian generator [4]

$$
\pi_{j}=p_{j}+i \pi \lambda / L \sum_{i \neq j} \cot \left(\pi\left(x_{i}-x_{j}\right) / L\right) P_{i j}
$$

where $p_{j}$ is the ordinary momentum operator, and showed that the Hamiltonian $H=\sum_{j} \pi_{j}^{2}$ is fully integrable and is same as the SCM up to a modification $\lambda\left(\lambda-P_{i j}\right)$ plus some trivial constant. The new operator $\pi_{j}$ is the momentum operator for the pseudo-particles, and the momenta $\sum_{j} k_{j}$ corresponds to the eigenvalues of $\sum_{j} \pi_{j}$. This new Hamiltonian should be considered as the model of one-dimensional "anyons" with fractional exchange statistics.

The single particle destruction operation on the ground state of $N+1$ "anyons" is, then, given by

$$
\Psi(x)|0\rangle_{N+1}=z^{-\lambda N / 2} \prod_{j=1}^{N}\left(z-z_{j}\right)^{\lambda} z_{j}^{-\lambda / 2}|0\rangle_{N},
$$

where $z=\exp (i 2 \pi x / L)$. Now, a similar technique used for the DDDCF can be employed to solve for the HPOGF. In this case, the contributing partitions have no more than $p$ rows and $q-1$ columns (i.e. $p$ quasi-holes and $q-1$ quasi-particles). Therefore, the "natural" exchange statistics of the real particles is fully compatible with the exclusion statistics of the elementary excitations.

In the thermodynamic limit the one-particle Green's function (hole propagator) is given by

$$
\left\langle 0\left|\Psi^{\dagger}(x, t) \Psi(0,0)\right| 0\right\rangle=\rho D e^{-i \pi \lambda \rho} \prod_{i=1}^{q-1}\left(\int_{0}^{\infty} d x_{i}\right) \prod_{j=1}^{p}\left(\int_{0}^{1} d y_{j}\right) F\left(q-1, p, \lambda \mid\left\{x_{i}, y_{j}\right\}\right) e^{i(Q x-E t)},
$$


where $F\left(q-1, p, \lambda \mid\left\{x_{i}, y_{j}\right\}\right)$ is given by Eq. (17) and D by

$$
D=\frac{\lambda^{2 p(q-1)} \Gamma(1+\lambda)}{\lambda^{p-q+1}(q-1) ! p !} A(q-1, p, \lambda)
$$

$\mathrm{Q}$ and $\mathrm{E}$ are same as before except for the number of $x_{j}$. At integer values of $\lambda$ (i.e. $q=1$ case where only quasi-holes are excited), based on the equal-time results of Forrester [23] Haldane made a conjecture [19] which agrees with this formula. I conjecture that the minimal form factor for any two-point correlation function is given by $F\left(m, n, \lambda \mid\left\{x_{i}, y_{j}\right\}\right)$ if the intermediate states involve only $m$ quasi-particles and $n$ quasi-holes.

In conclusion, the SCM is shown to possess the fractional exclusion and exchange statistics. The motifs representing the full spectrum are constructed and used to demonstrate the exclusion statistics, explicitly. The fractional statistics in the SCM is also confirmed by calculating the exact dynamical density-density correlation function and one-particle Green's function (hole propagator) at any rational interaction coupling constant using the theory of Jack symmetric orthogonal polynomial. The details of the calculation including the full Green's function will be published elsewhere.

While this Letter focuses on the fractional statistics aspect of the SCM, the method for calculating the correlation functions developed here could be of interest to a wide variety of people working on the disordered electronic system, the quantum chaos, the random matrix theory, 2D QCD, etc.

I thank F.D.M. Haldane, F. Wilczek, Y. M. Cho, T. Hwa, C. Johnson, R. Narayanan, and R. Kamien for useful discussions. This work is supported by DOE grant \#DE-FG0290ER40542. 


\section{REFERENCES}

[1] "Fractional Statistics and Anyon Superconductivity", ed. F. Wilczek, World Scientific (Singapore), 1990.

[2] F. D. M. Haldane, Phys. Rev. Lett. 67, 937 (1991).

[3] F. Calogero, J. Math. Phys. 10, 2191 (1969); B. Sutherland, Phys. Rev. A4, 2019 (1971); A5, 1372 (1972).

[4] F. D. M. Haldane, Z. N. C. Ha, J. C. Talstra, D. Bernard, and V. Pasquier, Phys. Rev. Lett. 69, 2021 (1992).

[5] R. P. Stanley, Adv. Math. 77, 76 (1989).

[6] J. Dyson, J. Math. Phys. 3, 140 (1962); 3, 157 (1962).

[7] B. D. Simons, P. A. Lee, and B. L. Altshuler, Phys. Rev. Lett. 70, 4122(1993); Nucl. Phys. B (to be published).

[8] F. D. M. Haldane and M. R. Zirnbauer, Phys. Rev. Lett. 71, 4055 (1993).

[9] M. R. Zirnbauer and F. D. M. Haldane, unpublished.

[10] J. Minahan, private communication.

[11] F. D. M. Haldane, in the proceeding of the International Colloquium in Modern Field Theory, Tata Institute, Bombay, India, January 5-12, 1994.

[12] C. N. Yang and C. P. Yang, J. Math. Phys. 10, 1115 (1969).

[13] B. Sutherland, in Lecture Notes in Physics 242, Springer-Verlag, 1985.

[14] This is also discussed in a recent preprint by D. Bernard and Y. S. Wu.

[15] Y. S. Wu, unpublished.

[16] I. G. Macdonald, "Symmetric Functions and Hall Polynomials," Oxford Univ. Press 
(New York), 1979.

[17] I. G. Macdonald, in Lecture Notes in Math. 1271, Springer-Verlag, 1987; For proofs, see K. W. J. Kadell, Compos. Math. 87, 5 (1993); Second edition of [16], to appear.

[18] P. J. Hanlon, R. P. Stanley, and J. R. Stembridge, Contemp. Math. 138, 151 (1992).

[19] F. D. M. Haldane, in the Proceedings of the 16th Taniguchi Symposium, Kashikojima, Japan, October 26-29, 1993, eds. A. Okiji and N. Kawakami, Springer-Verlag, 1994

[20] R. B. Laughlin, in The Quantum Hall Effect, Springer-Verlag, 1990.

[21] A. Lenard, J. Math. Phys. 5, 930 (1964).

[22] A. P. Polychronakos, Phys. Rev. Lett. 69, 703 (1992).

[23] P. J. Forrester, Phys. Lett. A179, 127(1993); Nucl. Phys. B388, 671 (1992). 